\title{
Hamiltonian structure of
}

\section{gauge-invariant variational}

\section{problems}

\author{
Marco Castrillón López ${ }^{1}$ and Jaime Muñoz Masqué2
}

${ }^{1}$ ICMAT(CSIC-UAM-UC3M-UCM), Departamento de Geometría y Topología, Facultad de Matemáticas, Universidad Complutense de Madrid, 28040-Madrid, Spain mcastri@mat.ucm.es

${ }^{2}$ Instituto de Física Aplicada, CSIC, C/Serrano 144, 28006-Madrid, Spain jaime@iec.csic.es

\begin{abstract}
Let $C \rightarrow M$ be the bundle of connections of a principal bundle on $M$. The solutions to Hamilton-Cartan equations for a gauge-invariant Lagrangian density $\Lambda$ on $C$ satisfying a weak condition of regularity, are shown to admit an affine fibre-bundle structure over the set of solutions to Euler-Lagrange equations for $\Lambda$. This structure is also studied for the Jacobi fields and for the moduli space of extremals.
\end{abstract}

\section{Introduction}

Let $p: E \rightarrow M$ be a fibred manifold and let $\Lambda$ be a Lagrangian density on $J^{1} E$. The solutions to the Hamilton-Cartan (H-C, in short) equations

e-print archive: http://lanl.arXiv.org/abs/1004.4923v1 
(e.g., see [7,8], or [14]) for $\Lambda$, are the sections $\bar{s}: M \rightarrow J^{1} E$ of the canonical projection $p_{1}: J^{1} E \rightarrow M$ fulfilling equation (3.1) below. If $\Lambda$ is regular, i.e., its Hessian metric is non-singular (cf. Definition 5.4 below), then every solution $\bar{s}$ to the $\mathrm{H}-\mathrm{C}$ equations is holonomic, i.e., $\bar{s}=j^{1} s$, and $s$ is a critical section for $\Lambda$, that is, a solution to the Euler-Lagrange (E-L, in short) equations. Nevertheless, a Lagrangian defined on the bundle of connections $p: C \rightarrow M$ of a principal bundle $\pi: P \rightarrow M$, which is invariant under the gauge group of $P$, is never regular. Therefore, Hamilton-Cartan equations and $\mathrm{E}-\mathrm{L}$ are not equivalent for all the gauge-invariant variational problems on connections.

In the present work we show that the solutions to $\mathrm{H}-\mathrm{C}$ equations for a gauge-invariant Lagrangian density $\Lambda$ on the bundle $C$ satisfying a weak condition of regularity (most of interesting gauge-invariant Lagrangians in the field theory satisfies this condition) admits an affine fibre-bundle structure over the set of critical sections of the variational problem defined by the density under consideration. Moreover, the structure of this fibration is completely determined; see Theorem 5.6 below. This proves, in particular, that the E-L equations of a gauge-invariant Lagrangian are essentially of first order.

Such a structure is meaningful even from the point of view of the observables of the field theory, because for every extremal section $s$ the curvature is constant along the fibre over $j^{1} s$.

By passing to the quotient such a fibre bundle modulo the gauge group, we obtain a - not necessary trivial - vector bundle associated to the principal bundle of the moduli of extremals of $\Lambda$; see Proposition 5.11 below. Finally, the aforementioned affine structure is also studied for the Jacobi fields; i.e., for the vector fields in the kernel of the linearization of the $\mathrm{E}-\mathrm{L}$ and $\mathrm{H}-\mathrm{C}$ operators at any extremal (cf. Theorem 5.12 below).

\section{Notations and preliminaries}

\section{$2.1 \quad$ Jet bundles}

The bundle of $r$-jets of a fibred manifold $p: E \rightarrow M$ is denoted by $p_{r}: J^{r}(p)$ $=J^{r} E \rightarrow M$ with projections $p_{r k}: J^{r} E \rightarrow J^{k} E, r \geq k$, the $r$-jet extension of a section $s: M \rightarrow E$ of $p$ being denoted by $j^{r} s$. Every $p$-fibred coordinate system $\left(x^{i}, y^{\alpha}\right), 1 \leq i \leq n=\operatorname{dim} M, 1 \leq \alpha \leq m=\operatorname{dim} E-n$, defined on an open subset $U \subseteq E$, induces a coordinate system $\left(x^{i}, y_{I}^{\alpha}\right), 0 \leq|I| \leq r$, on $\left(p_{r 0}\right)^{-1}(U)=J^{r} U$; namely, $y_{I}^{\alpha}\left(j_{x}^{r} s\right)=\left(\partial^{|I|}\left(y^{\alpha} \circ s\right) / \partial x^{I}\right)(x)$, with $y_{0}^{\alpha}=y^{\alpha}$. 
Every fibred map $\Phi: E \rightarrow E^{\prime}$ whose induced map $\phi: M \rightarrow M^{\prime}$ on the base manifold is a diffeomorphism, induces a map $\Phi^{(r)}: J^{r} E \rightarrow J^{r} E^{\prime}$ by setting, $\Phi^{(r)}\left(j_{x}^{r} s\right)=j_{\phi(x)}^{r}\left(\Phi \circ s \circ \phi^{-1}\right), \forall j_{x}^{r} s \in J^{r} E$.

An automorphism of $p$ is a pair of diffeomorphisms $\Phi: E \rightarrow E, \phi: M \rightarrow$ $M$ such that $p \circ \Phi=\phi \circ p$. The set of all automorphisms of $p$ is a group denoted by Aut $E$.

For every - not necessarily p-projectable - vector field $Y \in \mathfrak{X}(E)$ a unique vector field $Y^{(1)} \in \mathfrak{X}\left(J^{1} E\right)$ exists (called the one-jet prolongation of $Y$ ) such that, 1st) $Y^{(1)}$ is $p_{10}$-projectable onto $Y$, and 2nd) $L_{Y^{(1)}} \mathcal{C} \subseteq \mathcal{C}$, where $\mathcal{C}$ is the contact system; i.e., the differential system generated by the contact one-forms $\theta^{\alpha}=d y^{\alpha}-y_{i}^{\alpha} d x^{i}$ in $\Omega^{1}\left(J^{1} E\right)$.

If $Y$ is a $p$-vertical vector field (the only case that we consider below), then the formulas of one-jet prolongation are as follows (cf. [14, Section 2.4]):

$$
\begin{aligned}
Y^{(1)} & =v^{\alpha} \frac{\partial}{\partial y^{\alpha}}+v_{i}^{\alpha} \frac{\partial}{\partial y_{i}^{\alpha}}, \quad v_{i}^{\alpha}=\frac{\partial v^{\alpha}}{\partial x^{i}}+\frac{\partial v^{\alpha}}{\partial y^{\beta}} y_{i}^{\beta}, \\
Y & =v^{\alpha} \frac{\partial}{\partial y^{\alpha}}, \quad v^{\alpha} \in C^{\infty}(E) .
\end{aligned}
$$

Lemma $2.1([14])$. For every $p$-vertical vector field $Y \in \mathfrak{X}^{v}(E)$ the value of the vector field $Y^{(1)}$ at a point $j_{x}^{1} s \in J^{1} E$ depends only on $j_{x}^{1}(Y \circ s)$.

\subsection{Bundles of connections}

An automorphism of a principal $G$-bundle $\pi: P \rightarrow M$ is an equivariant diffeomorphism $\Phi: P \rightarrow P$; i.e., $\Phi(u \cdot g)=\Phi(u) \cdot g, \forall u \in P, \forall g \in G$. We denote by Aut $P$ the group of all automorphisms of $P$ under composition. Every $\Phi \in$ Aut $P$ determines a unique diffeomorphism $\phi: M \rightarrow M$, such that $\pi \circ \Phi=\phi \circ \pi$. If $\phi$ is the identity map, then $\Phi$ is said to be a gauge transformation (cf. $[1,3.2 .1]$ ). We denote by $\operatorname{Gau} P \subset \operatorname{Aut} P$ the subgroup of all gauge transformations.

A vector field $X \in \mathfrak{X}(P)$ is said to be $G$-invariant if $R_{g} \cdot X=X, \forall g \in G$. If $\Phi_{t}$ is the flow of a vector field $X \in \mathfrak{X}(P)$, then $X$ is $G$-invariant if and only if $\Phi_{t} \in \operatorname{Aut} P, \forall t \in \mathbb{R}$. Because of this we denote the Lie subalgebra of $G$-invariant vector fields on $P$ by aut $P \subset \mathfrak{X}(P)$. Each $G$-invariant vector field on $P$ is $\pi$-projectable. Similarly, a $\pi$-vertical vector field $X \in \mathfrak{X}(P)$ is $G$-invariant if and only if $\Phi_{t} \in \mathrm{Gau} P, \forall t \in \mathbb{R}$. We denote by gau $P \subset \operatorname{aut} P$ 
the ideal of all $\pi$-vertical $G$-invariant vector fields on $P$, which is called the gauge algebra of $P$.

The group $G$ acts on $T P$ by setting $X \cdot g=\left(R_{g}\right)_{*}(X), \forall X \in T P$, $\forall g \in G$. The global sections of the quotient vector bundle $T(P) / G$ can naturally be identified to aut $P$; i.e., aut $P \cong \Gamma(M, T(P) / G)$. Similarly, gau $P \cong$ $\Gamma(M, \operatorname{ad} P)$, where ad $P$ denotes the adjoint bundle: The bundle associated to $P$ by the adjoint representation of $G$ on its Lie algebra $\mathfrak{g}$, denoted by $\pi_{\mathfrak{g}}: \operatorname{ad} P \rightarrow M$; that is, $\operatorname{ad} P=(P \times \mathfrak{g}) / G$, where the action of $G$ on $P \times \mathfrak{g}$ is defined by

$$
(u, B) \cdot g=\left(u \cdot g, \operatorname{Ad}_{g^{-1}}(B)\right), \quad \forall u \in P, \forall B \in \mathfrak{g}, \forall g \in G
$$

The $G$-orbit in ad $P$ of a pair $(u, B) \in P \times \mathfrak{g}$ is denoted by $(u, B)_{\text {ad }}$.

An exact sequence of vector bundles over $M$ (the so-called Atiyah sequence) holds, $0 \rightarrow \operatorname{ad} P \rightarrow T(P) / G \stackrel{\pi_{*}}{\longrightarrow} T M \rightarrow 0$. The fibres $(\operatorname{ad} P)_{x}$ are endowed with a Lie-algebra structure determined by $\left[(u, B)_{\mathrm{ad}},\left(u, B^{\prime}\right)_{\mathrm{ad}}\right]=$ $\left(u,-\left[B, B^{\prime}\right]\right)_{\text {ad }}$, for all $u \in \pi^{-1}(x), B, B^{\prime} \in \mathfrak{g}$, where $[\cdot, \cdot \cdot]$ denotes the bracket in $\mathfrak{g}$. The sign of the bracket above is needed in order to ensure that the natural identification gau $P \cong \Gamma(M, \operatorname{ad} P)$ is a Lie-algebra isomorphism, when gau $P$ is considered as a Lie subalgebra of $\mathfrak{X}(P)$.

Let $X^{h_{\Gamma}} \in \mathfrak{X}(P)$ be the horizontal lift of a vector field $X \in \mathfrak{X}(M)$ with respect to a connection $\Gamma$ on $\pi: P \rightarrow M$. The vector field $X^{h_{\Gamma}}$ is $G$-invariant and projects onto $X$ (cf. [10, II. Proposition 1.2]). Hence we have a splitting of the Atiyah sequence, $s_{\Gamma}: T M \rightarrow T(P) / G, s_{\Gamma}(X)=X^{h_{\Gamma}}$. Conversely, any splitting $\sigma: T M \rightarrow T(P) / G$ of the Atiyah sequence (i.e., $s$ is a vector bundle homomorphism such that $\pi_{*} \circ s=\mathrm{id}_{T M}$ ) comes from a unique connection on $P$ so that there is a natural bijection between connections on $P$ and splittings of the Atiyah sequence. We thus define the bundle of connections $p: C=C(P) \rightarrow M$ as the sub-bundle of $\operatorname{Hom}(T M, T(P) / G)$ determined by all $\mathbb{R}$-linear mappings $\lambda: T_{x} M \rightarrow(T(P) / G)_{x}$ such that $\pi_{*} \circ \lambda=\mathrm{id}_{T_{x} M}$. Connections on $P$ can be identified to the global sections of $p: C \rightarrow M$. We also denote by $s_{\Gamma}: M \rightarrow C$ the section of the bundle of connections induced by $\Gamma$.

An element $\lambda: T_{x} M \rightarrow(T(P) / G)_{x}$ of the bundle $C$ over a point $x \in M$ is nothing but a 'connection at a point $x$ '; i.e., $\Lambda$ induces a complementary subspace $H_{u}$ of the vertical subspace $V_{u}(P) \subset T_{u}(P)$ for every $u \in \pi^{-1}(x)$. Any other connection at $x$ can be written as $\lambda^{\prime}=h+\lambda$, where $h: T_{x} M \rightarrow$ $(\operatorname{ad} P)_{x}$ is a linear map. Hence $C$ is an affine bundle modelled over the vector bundle $\operatorname{Hom}(T M, \operatorname{ad} P) \cong T^{*} M \otimes \operatorname{ad} P$. 
Let $\left(U ; x^{i}\right)$ be a coordinate open domain in $M$ such that $\pi^{-1}(U) \cong U \times G$. For every $B \in \mathfrak{g}$ we define a flow of gauge transformations over $U$ by setting $\varphi_{t}^{B}(x, g)=(x, \exp (t B) \cdot g), x \in U$. Let $\tilde{B}$ be the corresponding infinitesimal generator. If $\left(B_{1}, \ldots, B_{m}\right)$ is a basis of $\mathfrak{g}$, then $\tilde{B}_{1}, \ldots, \tilde{B}_{m}$ is a basis of $\Gamma(U, \operatorname{ad} P)$. Let $p: C \rightarrow M$ be the bundle of connections of $P$. The horizontal lift with respect to $\Gamma$ of the basic vector field $\partial / \partial x^{i}$ is given as follows:

$$
s_{\Gamma}\left(\frac{\partial}{\partial x^{i}}\right)=\left(\frac{\partial}{\partial x^{i}}\right)^{h_{\Gamma}}=\frac{\partial}{\partial x^{i}}-\left(A_{i}^{\alpha} \circ s_{\Gamma}\right) \tilde{B}_{\alpha} .
$$

The functions $\left(x^{i}, A_{j}^{\alpha}\right), i, j=1, \ldots, n=\operatorname{dim} M, 1 \leq \alpha \leq m=\operatorname{dim} G$, induce a coordinate system on $p^{-1}(U)=C\left(\pi^{-1} U\right)$ (cf. [2]).

Each automorphism $\Phi \in$ Aut $P$ acts on connections of $P$ by pulling back connection forms; that is, $\Gamma^{\prime}=\Phi(\Gamma)$ where $\omega_{\Gamma^{\prime}}=\left(\Phi^{-1}\right)^{*} \omega_{\Gamma}$ (cf. [10, II. Proposition 6.2-(b)]). If $\Psi \in$ Aut $P$ is another automorphism, then $(\Psi \circ \Phi)(\Gamma)=\Psi(\Phi(\Gamma))$. For each $\Phi \in$ Aut $P$ there exists a unique diffeomorphism $\Phi_{C}: C \rightarrow C$ such that $p \circ \Phi_{C}=\Phi \circ p$, where $\Phi: M \rightarrow M$ is the diffeomorphism induced by $\Phi$ on the base manifold. We thus obtain a group homomorphism Aut $P \rightarrow \operatorname{Diff} C$. For every connection $\Gamma$ on $P$ we have $\Phi_{C} \circ s_{\Gamma}=s_{\Phi(\Gamma)}$.

If $\Phi_{t}$ is the flow of a $G$-invariant vector field $X \in \operatorname{aut} P$, then $\left(\Phi_{t}\right)_{C}$ is a one-parameter group in Diff $C$ with infinitesimal generator denoted by $X_{C}$, and the map aut $P \rightarrow \mathfrak{X}(C), X \mapsto X_{C}$ is a Lie-algebra homomorphism.

\subsection{Affine-bundle structures}

Let $V(p)=\left\{X \in T E: p_{*} X=0\right\}$ be the vertical subbundle of a fibred manifold $p: E \rightarrow M$.

(a) Let $p: E \rightarrow M$ be an affine bundle modelled over the vector bundle $p_{W}: W \rightarrow M$. The directional derivative determines an isomorphism of vector bundles over $E, p^{*} W \cong V(p),(\mathrm{e}, w) \mapsto X_{e, w}, p(\mathrm{e})=$ $p_{W}(w)=x$, where $X_{e, w}$ is the tangent vector at $t=0$ to the curve $t \mapsto t w+\mathrm{e}$, which takes values in the fibre $p^{-1}(x)$. In coordinates, $X_{e, w}=w^{\alpha}(w)\left(\partial / \partial \mathrm{e}^{\alpha}\right)_{e}$.

(b) Moreover, if $p: E \rightarrow M$ is an arbitrary surjective submersion, then the projection $p_{10}: J^{1} E \rightarrow E$ is endowed with an affine-bundle structure modelled over $p^{*} T^{*} M \otimes V(p)$. In fact, every jet $j_{x}^{1} s \in\left(p_{10}\right)^{-1}(\mathrm{e})$, with $s(x)=\mathrm{e}$, can be identified to the section $s_{*, e}: T_{x} M \rightarrow T_{e} E$ of $p_{*, e}: T_{e} E \rightarrow T_{x} M$. Hence, if $j_{x}^{1} s^{\prime} \in\left(p_{10}\right)^{-1}(\mathrm{e})$ is another jet, then $p_{*, e} \circ$ 
$\left(s_{*, e}^{\prime}-s_{*, e}\right)=0$ and accordingly $s_{*, \mathrm{e}}^{\prime}-s_{*, \mathrm{e}}$ takes values into $V_{e}(p)$. Therefore, $s_{*, e}^{\prime}-s_{*, e}$ determines an element in $\operatorname{Hom}\left(T_{x} M \otimes V_{e}(p)\right)=$ $T_{x}^{*} M \otimes V_{e}(p)$. From (a) it follows an isomorphism, $p_{10}^{*}\left(p^{*} T^{*} M\right.$ $\otimes V(p))=T^{*} M \otimes_{J^{1} C} V(p) \cong V\left(p_{10}\right)$.

\subsection{The Hessian metric}

If $p: E \rightarrow M$ be an affine bundle modelled over the vector bundle $p_{W}: W \rightarrow$ $M$, then, according to the item (a) in Section 2.3, every $w \in\left(p_{W}\right)^{-1}(x)$ induces a vector field along the fibre $X_{w} \in \mathfrak{X}\left(p^{-1}(x)\right), \quad X_{w}(\mathrm{e})=X_{e, w}$, $\forall \mathrm{e} \in p^{-1}(x)$. For every $f \in C^{\infty}(E)$ and every e $\in E$, with $x=p(\mathrm{e})$, a bilinear form

$$
\operatorname{Hess}_{e}(f): V_{e}(p) \times V_{e}(p) \rightarrow \mathbb{R}
$$

can be defined as follows: $\operatorname{Hess}_{e}(f)\left(w_{1}, w_{2}\right)=X_{w_{2}}(\mathrm{e})\left(\left(d_{E / M} f\right) X_{w_{1}}\right)$, where the canonical isomorphism $W_{x} \cong V_{e}(p)$, defined in the item (a) in Section 2.3, has be used and $d_{E / M}$ denotes the fibred derivative, e.g., see [8]. As this form is proved to be symmetric, $\mathrm{e} \mapsto \operatorname{Hess}_{e}(f)$ defines a section of the vector bundle $S^{2} V^{*}(p) \cong p^{*} S^{2} W^{*}$.

\section{$3 \mathrm{H}-\mathrm{C}$ equations}

Let $p: E \rightarrow M$ be a fibred manifold, $\operatorname{dim} M=n, \operatorname{dim} E=m+n$, where $M$ is assumed to be connected and oriented by a volume form $\mathbf{v}$. Below, Latin indices run from 1 to $n$, and Greek indices run from 1 to $m$. The solutions to the Hamilton-Cartan equations for a density $\Lambda=L \mathbf{v}, L \in C^{\infty}\left(J^{1} E\right)$ on $p$, are the sections $\bar{s}: M \rightarrow J^{1} E$ of the canonical projection $p_{1}: J^{1} E \rightarrow M$ such that,

$$
\bar{s}^{*}\left(i_{X} d \Theta_{\Lambda}\right)=0, \quad \forall X \in \mathfrak{X}^{v}\left(J^{1} E\right),
$$

where

(i): $\Theta_{\Lambda}=(-1)^{i-1}\left(\partial L / \partial y_{i}^{\alpha}\right) \theta^{\alpha} \wedge \mathbf{v}_{i}+L \mathbf{v}$ is the Poincaré-Cartan form attached to $\Lambda$ (cf. [8], [14]);

(ii): $\mathfrak{X}^{v}\left(J^{1} E\right)$ denotes the Lie algebra of $p_{1}$-vertical vector fields;

(iii): $\theta^{\alpha}=d y^{\alpha}-y_{i}^{\alpha} d x^{i}$ are the standard contact forms on the one-jet bundle; and 
(iv): $\left(x^{i}, y^{\alpha}, y_{i}^{\alpha}\right)$ is the coordinate system on $J^{1} E$ induced by a fibred coordinate system $\left(x^{i}, y^{\alpha}\right)$ for the submersion $p$, adapted to the given volume form; i.e., $\mathbf{v}=d x^{1} \wedge \cdots \wedge d x^{n}$ and $\mathbf{v}_{i}=(-1)^{i-1} i_{\partial / \partial x^{i}} \mathbf{v}$.

Lemma 3.1. A section $\bar{s}: M \rightarrow J^{1} E$ of $p_{1}: J^{1} E \rightarrow M$ is a solution to the $H-C$ equations (3.1) if and only if the following equations hold:

$$
\begin{gathered}
\left(s_{j}^{\beta}-\bar{s}_{j}^{\beta}\right)\left(\frac{\partial^{2} L}{\partial y_{i}^{\alpha} \partial y_{j}^{\beta}} \circ \bar{s}\right)=0, \quad 1 \leq i \leq n, 1 \leq \alpha \leq m, \\
-\frac{\partial}{\partial x^{j}}\left(\frac{\partial L}{\partial y_{j}^{\alpha}} \circ \bar{s}\right)+\frac{\partial L}{\partial y^{\alpha}} \circ \bar{s}+\left(s_{j}^{\beta}-\bar{s}_{j}^{\beta}\right)\left(\frac{\partial^{2} L}{\partial y^{\alpha} \partial y_{j}^{\beta}} \circ \bar{s}\right)=0,
\end{gathered}
$$

for $1 \leq \alpha \leq m$, where $s^{\alpha}=y^{\alpha} \circ \bar{s}, s_{i}^{\alpha}=\partial s^{\alpha} / \partial x^{i}$, and $\bar{s}_{i}^{\alpha}=y_{i}^{\alpha} \circ \bar{s}$.

Proof. As a simple computation shows, we have

$$
d \Theta_{\Lambda}=\theta^{\beta} \wedge\left((-1)^{j} d\left(\frac{\partial L}{\partial y_{j}^{\beta}}\right) \wedge \mathbf{v}_{j}+\frac{\partial L}{\partial y^{\beta}} \mathbf{v}\right) .
$$

Hence

$$
\begin{aligned}
\bar{s}^{*}\left(i_{\partial / \partial y_{i}^{\alpha}} d \Theta_{\Lambda}\right)= & (-1)^{j-1}\left(\frac{\partial^{2} L}{\partial y_{i}^{\alpha} \partial y_{j}^{\beta}} \circ \bar{s}\right) \bar{s}^{*} \theta^{\beta} \wedge \mathbf{v}_{j} \\
\bar{s}^{*}\left(i_{\partial / \partial y^{\alpha}} d \Theta_{\Lambda}\right)= & \left(-\frac{\partial}{\partial x^{j}}\left(\frac{\partial L}{\partial y_{j}^{\alpha}} \circ \bar{s}\right)+\frac{\partial L}{\partial y^{\alpha}} \circ \bar{s}\right) \mathbf{v} \\
& +(-1)^{j-1}\left(\frac{\partial^{2} L}{\partial y^{\alpha} \partial y_{j}^{\beta}} \circ \bar{s}\right) \bar{s}^{*} \theta^{\beta} \wedge \mathbf{v}_{j}
\end{aligned}
$$

and the formulas in the statement follow.

If $\bar{s}=j^{1} s$ is a holonomic section, then $\bar{s}$ is a solution to the H-C equations if and only if $s$ is a solution to the $\mathrm{E}-\mathrm{L}$ equations. If $L$ is regular, then the converse holds true: Every solution to the $\mathrm{H}-\mathrm{C}$ equations, is of the form $\bar{s}=j^{1} s, s$ being a solution to the E-L equations. Hence, for regular variational problems, $\mathrm{H}-\mathrm{C}$ equations are equivalent to $\mathrm{E}-\mathrm{L}$ equations; but this is no longer true for non-regular densities, as is the case for the Yang-Mills Lagrangian. 


\section{Jacobi fields}

\subsection{Jacobi fields introduced}

Let $p: E \rightarrow M$ be a fibred manifold and let $\Omega^{1}(E / M)=\Gamma\left(M, V^{*}(p)\right)$. Let

$$
\mathcal{E}^{\Lambda}: \Gamma(p) \rightarrow \Omega^{1}(E / M) \otimes_{C^{\infty}(M)} \Omega^{n}(M)
$$

be the E-L operator of $\Lambda=L \mathbf{v}, L \in C^{\infty}\left(J^{1} E\right)$, which is the second-order differential operator locally given on a fibred coordinate system $\left(U ; x^{i}, y^{\alpha}\right)$ for the submersion $p$ and for every section $s$ of $\left.p\right|_{U}$ by,

$$
\mathcal{E}^{\Lambda}(s)=\left(\mathcal{E}_{\alpha}^{\Lambda} \circ j^{2} s\right) d_{E / M} y^{\alpha} \otimes \mathbf{v},
$$

where the functions $\mathcal{E}_{\alpha}^{\Lambda} \in C^{\infty}\left(J^{2} E\right)$ are defined as follows:

$$
\mathcal{E}_{\alpha}^{\Lambda}\left(j_{x}^{2} s\right)=\frac{\partial L}{\partial y^{\alpha}}\left(j_{x}^{1} s\right)-\frac{\partial}{\partial x^{j}}\left(\frac{\partial L}{\partial y_{j}^{\alpha}} \circ j^{1} s\right)(x) .
$$

The linearization of $\mathcal{E}^{\Lambda}$ at $s \in \Gamma(p)$ is the operator

$$
\mathcal{L}_{s} \mathcal{E}^{\Lambda}: T_{s} \Gamma(p)=\Gamma\left(M, s^{*} V(p)\right) \rightarrow \Omega^{1}(E / M) \otimes_{C^{\infty}(M)} \Omega^{n}(M)
$$

defined as follows.

If $S:(-\varepsilon, \varepsilon) \times U \rightarrow E$ is a one-parameter family of sections, i.e., $p \circ S_{t}=$ $\operatorname{id}_{U},|t|<\varepsilon$, then a vector field $X \in \Gamma\left(U, s^{*} V(p)\right)$ along $s=S_{0}$ - called the 'initial velocity' of $S$ - is defined to be the tangent vector $X(x) \in V_{s(x)}(p)$ at $t=0$ to the curve $t \mapsto S_{t}(x)$, which takes values in the fibre $p^{-1}(x)$ for every point $x \in U$. Expanding $y^{\alpha} \circ S_{t}$ up to second order, we obtain $y^{\alpha} \circ S_{t}=$ $y^{\alpha} \circ s+t v^{\alpha}+t^{2} f^{\alpha}$, for certain functions $v^{\alpha} \in C^{\infty}(U), f^{\alpha} \in C^{\infty}((-\varepsilon, \varepsilon) \times$ $U)$. Hence,

$$
X(x)=v^{\alpha}(x)\left(\partial / \partial y^{\alpha}\right)_{s(x)}, \quad \forall x \in U
$$

Therefore, every vector field $X \in \Gamma\left(U, s^{*} V(p)\right)$ is the initial velocity of a one-parameter family of sections $S$, and we define,

$$
\mathcal{L}_{s} \mathcal{E}^{\Lambda}(X)=\left.\frac{\partial}{\partial t}\right|_{t=0} \mathcal{E}^{\Lambda}\left(S_{t}\right)
$$


The definition makes sense as it does not depend on the particular oneparameter family of sections chosen. In fact,

$$
\begin{aligned}
\left.\frac{\partial}{\partial t}\right|_{t=0}\left(\mathcal{E}_{\alpha}^{\Lambda} \circ j^{2} S_{t}\right)= & \left(\frac{\partial \mathcal{E}_{\alpha}^{\Lambda}}{\partial y^{\beta}} \circ j^{2} s\right) v^{\beta}+\left(\frac{\partial \mathcal{E}_{\alpha}^{\Lambda}}{\partial y_{j}^{\beta}} \circ j^{2} s\right) \frac{\partial v^{\beta}}{\partial x^{j}} \\
& +\left(\frac{\partial \mathcal{E}_{\alpha}^{\Lambda}}{\partial y_{j k}^{\beta}} \circ j^{2} s\right) \frac{\partial^{2} v^{\beta}}{\partial x^{j} \partial x^{k}} .
\end{aligned}
$$

This expression also shows that $\mathcal{L}_{s} \mathcal{E}^{\Lambda}(X)$ depends linearly on $X$.

Definition 4.1. A vector field $X \in \Gamma\left(M, s^{*} V(p)\right)$ defined along an extremal $s$ of a Lagrangian density $\Lambda$ on $p: E \rightarrow M$ is said to be a Jacobi field if $X \in \operatorname{ker} \mathcal{L}_{s} \mathcal{E}^{\Lambda}$.

Proposition 4.2. A vector field $X \in \Gamma\left(M, s^{*} V(p)\right)$ defined along an extremal $s$ of a Lagrangian density $\Lambda$ on $p: E \rightarrow M$ is a Jacobi field if and only if the following equation holds:

$$
\left(j^{1} s\right)^{*}\left(i_{Y} L_{\tilde{X}^{(1)}} d \Theta_{\Lambda}\right)=0, \quad \forall Y \in \mathfrak{X}^{v}\left(J^{1} E\right)
$$

where $\tilde{X} \in \mathfrak{X}^{v}(E)$ is an arbitrary p-vertical extension of $X$.

Proof. Equation (4.1) does not depend on the vertical extension chosen. In fact,

$$
\left(j^{1} s\right)^{*}\left(i_{Y} L_{\tilde{X}^{(1)}} d \Theta_{\Lambda}\right)=\left(j^{1} s\right)^{*}\left(L_{\tilde{X}^{(1)}}\left(i_{Y} d \Theta_{\Lambda}\right)\right)+\left(j^{1} s\right)^{*}\left(i_{\left[Y, \tilde{X}^{(1)}\right]} d \Theta_{\Lambda}\right),
$$

and the second term on the right-hand side vanishes, as $s$ is an extremal. Hence

$$
\left(j^{1} s\right)^{*}\left(i_{Y} L_{\tilde{X}^{(1)}} d \Theta_{\Lambda}\right)=d\left(\left(j^{1} s\right)^{*}\left(i_{\tilde{X}^{(1)}} i_{Y} d \Theta_{\Lambda}\right)\right)+\left(j^{1} s\right)^{*}\left(i_{\tilde{X}^{(1)}} d\left(i_{Y} \Theta_{\Lambda}\right)\right),
$$

and we conclude by simply applying Lemma 2.1.

Moreover, from the formula (3.2) the following identity is obtained:

$$
d \Theta_{\Lambda}=\mathcal{E}_{\alpha}^{\Lambda} \theta^{\alpha} \wedge \mathbf{v}+(-1)^{i} \frac{\partial^{2} L}{\partial y_{i}^{\alpha} \partial y^{\beta}} \theta^{\alpha} \wedge \theta^{\beta} \wedge \mathbf{v}_{i}+(-1)^{i} \frac{\partial^{2} L}{\partial y_{i}^{\alpha} \partial y_{j}^{\beta}} \theta^{\alpha} \wedge \theta_{j}^{\beta} \wedge \mathbf{v}_{i}
$$


where $\theta_{j}^{\beta}=d y_{j}^{\beta}-y_{(j k)}^{\beta} d x^{k}$. If $\tilde{\Phi}_{t}$ is the flow of $\tilde{X}$, then $j^{1} S_{t}=J^{1}\left(\tilde{\Phi}_{t}\right) \circ j^{1} s$ and from the previous formula we have

$$
\begin{aligned}
\left(j^{1} s\right)^{*}\left\{J^{1}\left(\tilde{\Phi}_{t}\right)^{*}\left(i_{Y} d \Theta_{\Lambda}\right)\right\} & =\left(j^{1} S_{t}\right)^{*}\left(i_{Y} d \Theta_{\Lambda}\right) \\
& =\left(\mathcal{E}_{\alpha}^{\Lambda} \circ j^{2} S_{t}\right) \theta^{\alpha}(Y) \mathbf{v} .
\end{aligned}
$$

Taking derivatives with respect to $t$ at $t=0$ in this formula, we have

$$
\left(j^{1} s\right)^{*}\left(L_{\tilde{X}^{(1)}}\left(i_{Y} d \Theta_{\Lambda}\right)\right)=\mathcal{L}_{s} \mathcal{E}^{\Lambda}(X) \mathbf{v},
$$

and we can conclude by simply applying formula (4.2) recalling that $s$ is an extremal.

Let $\mathcal{S}_{\Lambda}$ (resp. $\overline{\mathcal{S}}_{\Lambda}$ ) denote the set of solutions to the E-L equations (resp. $\mathrm{H}-\mathrm{C}$ equations) attached to a Lagrangian density $\Lambda=L \mathbf{v}, L \in C^{\infty}\left(J^{1} E\right)$.

Remark 4.3. If $S_{t} \in \mathcal{S}_{\Lambda}$ is a one-parameter family of extremals, then its initial velocity $X$ is readily seen to be a Jacobi field along the extremal $s=S_{0}$; in this case, $X$ is said to be 'integrable' (e.g., see [11, Definition 1.2], [12, Section 2.6]). Although important examples of non-integrable Jacobi fields exist, usually Jacobi fields along $s$ are considered as the tangent space $T_{s} \mathcal{S}_{\Lambda}$ at an extremal $s \in \mathcal{S}_{\Lambda}$ to the 'manifold' of solutions to the E-L equations for $\Lambda$. By the same token, we give the following

Definition 4.4. The tangent space $T_{\bar{s}} \overline{\mathcal{S}}_{\Lambda}$ at a solution $\bar{s} \in \overline{\mathcal{S}}_{\Lambda}$ to the 'manifold' of solutions to the $\mathrm{H}-\mathrm{C}$ equations for $\Lambda$ is defined to be the space of vector fields $\bar{X} \in \Gamma\left(M, \bar{s}^{*} V\left(p_{1}\right)\right)$ that satisfy the equation

$$
\bar{s}^{*}\left(i_{Y} L_{\tilde{X}} d \Theta_{\Lambda}\right)=0, \quad \forall Y \in \mathfrak{X}^{v}\left(J^{1} E\right),
$$

where $\tilde{X} \in \mathfrak{X}^{v}\left(J^{1} E\right)$ is a $p_{1}$-vertical extension of $\bar{X}$.

\subsection{The embedding $T_{s} \mathcal{S}_{\Lambda} \hookrightarrow T_{j^{1} s} \overline{\mathcal{S}}_{\Lambda}$}

Proposition 4.5. For every $s \in \mathcal{S}_{\Lambda}$, there is an embedding

$$
\begin{aligned}
T_{s} \mathcal{S}_{\Lambda} & \hookrightarrow T_{j^{1} s} \overline{\mathcal{S}}_{\Lambda}, \\
X & \mapsto \tilde{X}^{(1)} \circ j^{1} s,
\end{aligned}
$$

where $\tilde{X}$ is any p-vertical extension of $X \in \Gamma\left(M, s^{*} V(p)\right)$ to $E$. If $\Lambda$ is regular, then $T_{s} \mathcal{S}_{\Lambda} \cong T_{j^{1}} \overline{\mathcal{S}}_{\Lambda}, \forall s \in \mathcal{S}_{\Lambda}$. 
Proof. As a straightforward — but rather long - computation shows, a vector field $\bar{X} \in \Gamma\left(M, \bar{s}^{*} V\left(p_{1}\right)\right)$ with local expression

$$
\bar{X}=\left.v^{\alpha} \frac{\partial}{\partial y^{\alpha}}\right|_{\bar{s}}+\left.v_{i}^{\alpha} \frac{\partial}{\partial y_{i}^{\alpha}}\right|_{\bar{s}}, \quad v^{\alpha}, v_{i}^{\alpha} \in C^{\infty}(M),
$$

belongs to $T_{\bar{s}} \overline{\mathcal{S}}_{\Lambda}$ if and only if the following two equations hold:

$$
\begin{aligned}
0= & \left(\frac{\partial^{2} L}{\partial y^{\alpha} \partial y^{\sigma}} \circ \bar{s}-\frac{\partial^{3} L}{\partial x^{i} \partial y^{\alpha} \partial y_{i}^{\sigma}} \circ \bar{s}+\left(\frac{\partial s^{\beta}}{\partial x^{i}}-\bar{s}_{i}^{\beta}\right)\left(\frac{\partial^{3} L}{\partial y^{\alpha} \partial y^{\sigma} \partial y_{i}^{\beta}} \circ \bar{s}\right)\right. \\
& \left.-\frac{\partial s^{\gamma}}{\partial x^{j}}\left(\frac{\partial^{3} L}{\partial y^{\alpha} \partial y^{\gamma} \partial y_{j}^{\sigma}} \circ \bar{s}\right)-\frac{\partial \bar{s}_{h}^{\gamma}}{\partial x^{j}}\left(\frac{\partial^{3} L}{\partial y^{\alpha} \partial y_{h}^{\gamma} \partial y_{j}^{\sigma}} \circ \bar{s}\right)\right) v^{\alpha} \\
& +\left(\frac{\partial^{2} L}{\partial y^{\sigma} \partial y_{i}^{\alpha}} \circ \bar{s}-\frac{\partial^{2} L}{\partial y^{\alpha} \partial y_{i}^{\sigma}} \circ \bar{s}\right) \frac{\partial v^{\alpha}}{\partial x^{i}}-\left(\frac{\partial^{2} L}{\partial y_{j}^{\alpha} \partial y_{i}^{\sigma}} \circ \bar{s}\right) \frac{\partial v_{j}^{\alpha}}{\partial x^{i}} \\
& +\left(\left(\frac{\partial s^{\beta}}{\partial x^{j}}-\bar{s}_{j}^{\beta}\right)\left(\frac{\partial^{3} L}{\partial y^{\sigma} \partial y_{i}^{\alpha} \partial y_{j}^{\beta}} \circ \bar{s}\right)-\frac{\partial^{3} L}{\partial x^{j} \partial y_{i}^{\alpha} \partial y_{j}^{\sigma}} \circ \bar{s}\right. \\
& \left.-\frac{\partial s^{\beta}}{\partial x^{j}}\left(\frac{\partial^{3} L}{\partial y^{\beta} \partial y_{i}^{\alpha} \partial y_{j}^{\sigma}} \circ \bar{s}\right)-\frac{\partial \bar{s}_{h}^{\beta}}{\partial x^{j}}\left(\frac{\partial^{3} L}{\partial y_{h}^{\beta} \partial y_{i}^{\alpha} \partial y_{j}^{\sigma}} \circ \bar{s}\right)\right) v_{i}^{\alpha}, \\
0= & \left(\frac{\partial^{2} L}{\partial y_{i}^{\alpha} \partial y_{j}^{\sigma}} \circ \bar{s}\right)\left(\frac{\partial v^{\alpha}}{\partial x^{i}}-v_{i}^{\alpha}\right)+\left(\frac{\partial s^{\alpha}}{\partial x^{i}}-\bar{s}_{i}^{\alpha}\right)\left(\frac{\partial^{3} L}{\partial y^{\beta} \partial y_{i}^{\alpha} \partial y_{j}^{\sigma}} \circ \bar{s}\right) v^{\beta} \\
& +\left(\frac{\partial s^{\alpha}}{\partial x^{i}}-\bar{s}_{i}^{\alpha}\right)\left(\frac{\partial^{3} L}{\partial y_{i}^{\alpha} \partial y_{k}^{\beta} \partial y_{j}^{\sigma}} \circ \bar{s}\right) v_{k}^{\beta},
\end{aligned}
$$

where $s=p_{10} \circ \bar{s}, s^{\alpha}=y^{\alpha} \circ \bar{s}=y^{\alpha} \circ s$, and $\bar{s}_{i}^{\alpha}=y_{i}^{\alpha} \circ \bar{s}$. Along a holonomic section $\bar{s}=j^{1} s$, equations (4.3) and (4.4) become, respectively,

$$
\begin{aligned}
0= & \left(\frac{\partial^{2} L}{\partial y^{\alpha} \partial y^{\sigma}} \circ j^{1} s-\frac{\partial^{3} L}{\partial x^{i} \partial y^{\alpha} \partial y_{i}^{\sigma}} \circ j^{1} s-\frac{\partial s^{\gamma}}{\partial x^{j}}\left(\frac{\partial^{3} L}{\partial y^{\alpha} \partial y^{\gamma} \partial y_{j}^{\sigma}} \circ j^{1} s\right)\right. \\
& \left.-\frac{\partial^{2} s^{\gamma}}{\partial x^{h} \partial x^{j}}\left(\frac{\partial^{3} L}{\partial y^{\alpha} \partial y_{h}^{\gamma} \partial y_{j}^{\sigma}} \circ j^{1} s\right)\right) v^{\alpha} \\
& +\left(\frac{\partial^{2} L}{\partial y^{\sigma} \partial y_{i}^{\alpha}} \circ j^{1} s-\frac{\partial^{2} L}{\partial y^{\alpha} \partial y_{i}^{\sigma}} \circ j^{1} s\right) \frac{\partial v^{\alpha}}{\partial x^{i}}-\left(\frac{\partial^{2} L}{\partial y_{j}^{\alpha} \partial y_{i}^{\sigma}} \circ j^{1} s\right) \frac{\partial v_{j}^{\alpha}}{\partial x^{i}}
\end{aligned}
$$




$$
\begin{aligned}
& -\left(\frac{\partial^{3} L}{\partial x^{j} \partial y_{i}^{\alpha} \partial y_{j}^{\sigma}} \circ j^{1} s+\frac{\partial s^{\beta}}{\partial x^{j}}\left(\frac{\partial^{3} L}{\partial y^{\beta} \partial y_{i}^{\alpha} \partial y_{j}^{\sigma}} \circ j^{1} s\right)\right. \\
& \left.+\frac{\partial^{2} s^{\beta}}{\partial x^{h} \partial x^{j}}\left(\frac{\partial^{3} L}{\partial y_{i}^{\alpha} \partial y_{h}^{\beta} \partial y_{j}^{\sigma}} \circ j^{1} s\right)\right) v_{i}^{\alpha}, \\
0= & \left(\frac{\partial^{2} L}{\partial y_{i}^{\alpha} \partial y_{j}^{\sigma}} \circ j^{1} s\right)\left(\frac{\partial v^{\alpha}}{\partial x^{i}}-v_{i}^{\alpha}\right) .
\end{aligned}
$$

In addition, if $L$ is regular, then equation (4.6) is equivalent to saying that $v_{i}^{\alpha}=\partial v^{\alpha} / \partial x^{i}$, and from formula (2.1) and Lemma 2.1 we conclude that $\bar{X}$ is the one-jet prolongation of a Jacobi field, i.e., $\bar{X}=X^{(1)}$.

Remark 4.6. From formula (4.5) we deduce that a vector field

$$
\begin{aligned}
& X \in \Gamma\left(M, s^{*} V(p)\right), \\
& X=\left.v^{\alpha} \frac{\partial}{\partial y^{\alpha}}\right|_{s}, \quad v^{\alpha} \in C^{\infty}(M),
\end{aligned}
$$

belongs to $T_{s} \mathcal{S}_{\Lambda}$ if and only if the following equations hold:

$$
\begin{aligned}
0= & \left(\frac{\partial^{2} L}{\partial y^{\alpha} \partial y^{\sigma}} \circ j^{1} s-\frac{\partial^{3} L}{\partial x^{i} \partial y^{\alpha} \partial y_{i}^{\sigma}} \circ j^{1} s-\frac{\partial s^{\gamma}}{\partial x^{j}}\left(\frac{\partial^{3} L}{\partial y^{\alpha} \partial y^{\gamma} \partial y_{j}^{\sigma}} \circ j^{1} s\right)\right. \\
& \left.-\frac{\partial^{2} s^{\gamma}}{\partial x^{h} \partial x^{j}}\left(\frac{\partial^{3} L}{\partial y^{\alpha} \partial y_{h}^{\gamma} \partial y_{j}^{\sigma}} \circ j^{1} s\right)\right) v^{\alpha} \\
& +\left(\frac{\partial^{2} L}{\partial y^{\sigma} \partial y_{i}^{\alpha}} \circ j^{1} s-\frac{\partial^{2} L}{\partial y^{\alpha} \partial y_{i}^{\sigma}} \circ j^{1} s-\frac{\partial^{3} L}{\partial x^{j} \partial y_{i}^{\alpha} \partial y_{j}^{\sigma}} \circ j^{1} s\right. \\
& \left.-\frac{\partial s^{\beta}}{\partial x^{j}}\left(\frac{\partial^{3} L}{\partial y^{\beta} \partial y_{i}^{\alpha} \partial y_{j}^{\sigma}} \circ j^{1} s\right)-\frac{\partial^{2} s^{\beta}}{\partial x^{h} \partial x^{j}}\left(\frac{\partial^{3} L}{\partial y_{i}^{\alpha} \partial y_{h}^{\beta} \partial y_{j}^{\sigma}} \circ j^{1} s\right)\right) \frac{\partial v^{\alpha}}{\partial x^{i}} \\
& -\left(\frac{\partial^{2} L}{\partial y_{j}^{\alpha} \partial y_{i}^{\sigma}} \circ j^{1} s\right) \frac{\partial^{2} v^{\alpha}}{\partial x^{i} \partial x^{j}} .
\end{aligned}
$$

\section{$5 \quad \mathrm{H}-\mathrm{C}$ equations and gauge invariance}

\subsection{Gauge-invariant Lagrangians}

Definition 5.1. A smooth function $L: J^{1} C \rightarrow \mathbb{R}$ is said to be gauge invariant if $L \circ \Phi_{C}^{(1)}=L$ for every $\Phi \in \operatorname{Gau} P$. 
This equation obviously implies $X_{C}^{(1)} L=0$ for every $X \in \operatorname{gau} P$. The converse also holds if the group $G$ is connected. As every $\Phi \in \mathrm{Gau} P$ induces the identity map on $M$, the function $L$ is gauge invariant if and only if the gauge group is a group of symmetries for the Lagrangian density $\Lambda=L \mathbf{v}$, where $\mathbf{v}$ is an arbitrary volume form on the base manifold.

Let

$$
\begin{aligned}
& \Omega: J^{1} C \rightarrow \bigwedge^{2} T^{*} M \otimes \operatorname{ad} P, \\
& \Omega\left(j_{x}^{1} \sigma_{\Gamma}\right)=\left(\Omega_{\Gamma}\right)_{x},
\end{aligned}
$$

be the curvature map. Here, the curvature form $\Omega_{\Gamma}$ of the connection $\Gamma$ corresponding to a section $s_{\Gamma}$ of $p$, is seen to be a two form on $M$ with values in the adjoint bundle ad $P$. On the vector bundle $\wedge^{2} T^{*} M \otimes \operatorname{ad} P$ we consider the coordinate systems $\left(x^{i} ; R_{j k}^{\alpha}\right), j<k$, induced by a coordinate system $\left(U ; x^{i}\right)$ on $M$, and a basis $\left(B_{\alpha}\right)$ of $\mathfrak{g}$, as follows:

$$
\eta_{2}=\sum_{j<k}\left(R_{j k}^{\alpha}\left(\eta_{2}\right) d x^{j} \wedge d x^{k} \otimes \tilde{B}_{\alpha}\right)_{x}, \quad \forall \eta_{2} \in \bigwedge^{2} T_{x}^{*} M \otimes(\operatorname{ad} P)_{x} .
$$

The geometric formulation of Utiyama's Theorem (e.g., see [1]) states that a Lagrangian $L: J^{1} C \rightarrow \mathbb{R}$ is gauge invariant if and only $L$ factors through $\Omega$ as $L=\tilde{L} \circ \Omega$, where

$$
\tilde{L}: \bigwedge^{2} T^{*} M \otimes \operatorname{ad} P \rightarrow \mathbb{R}
$$

is a differentiable function which is invariant under the adjoint representation of $G$ on the curvature bundle. As the curvature map (5.1) is surjective, the function $\tilde{L}$ is unique.

\section{$5.2 \quad$ Projecting $\overline{\mathcal{S}}_{\Lambda}$ onto $\mathcal{S}_{\Lambda}$}

From Lemma 3.1 in Section 3, we readily obtain

Proposition 5.2. The $H-C$ equations of a Lagrangian $L$ on the bundle of connections $p: C \rightarrow M$ of a principal bundle $\pi: P \rightarrow M$, read as follows:

$$
\begin{gathered}
\left(s_{h, j}^{\beta}-\bar{s}_{h, j}^{\beta}\right)\left(\frac{\partial^{2} L}{\partial A_{i, k}^{\alpha} \partial A_{h, j}^{\beta}} \circ \bar{s}\right)=0, \quad \forall \alpha, i, k \\
-\frac{\partial}{\partial x^{j}}\left(\frac{\partial L}{\partial A_{i, j}^{\alpha}} \circ \bar{s}\right)+\frac{\partial L}{\partial A_{i}^{\alpha}} \circ \bar{s}+\left(s_{h, j}^{\beta}-\bar{s}_{h, j}^{\beta}\right)\left(\frac{\partial^{2} L}{\partial A_{i}^{\alpha} \partial A_{h, j}^{\beta}} \circ \bar{s}\right)=0, \quad \forall \alpha, i,
\end{gathered}
$$


where $\bar{s}: M \rightarrow J^{1} C$ is a section of $p_{1}: J^{1} C \rightarrow M$, and we have set

$$
s_{i}^{\alpha}=A_{i}^{\alpha} \circ p_{10} \circ \bar{s}, \quad s_{i, j}^{\alpha}=\frac{\partial s_{i}^{\alpha}}{\partial x^{j}}, \quad \bar{s}_{i, j}^{\alpha}=A_{i, j}^{\alpha} \circ \bar{s} .
$$

Lemma 5.3. The Hessian metric of a gauge-invariant Lagrangian $L$ on the bundle of connections $p: C \rightarrow M$ of a principal bundle $\pi: P \rightarrow M$, is singular; i.e.,

$$
\operatorname{det}\left(\frac{\partial^{2} L}{\partial A_{i, j}^{\alpha} \partial A_{k, l}^{\beta}}\right)_{\beta, k, l}^{\alpha, i, j}=0
$$

Proof. As $L=\tilde{L} \circ \Omega$, we have $\partial L / \partial A_{i, i}^{\alpha}=0$, taking the curvature equations into account, i.e.,

$$
R_{i j}^{\alpha} \circ \Omega=A_{i, j}^{\alpha}-A_{j, i}^{\alpha}-c_{\beta \gamma}^{\alpha} A_{i}^{\beta} A_{j}^{\gamma} .
$$

Hence, $\partial^{2} L / \partial A_{i, i}^{\alpha} \partial A_{k, l}^{\beta}=0$, for all indices $\beta, k, l$.

Definition 5.4. A Lagrangian $L \in C^{\infty}\left(J^{1} C\right)$ is said to be regular if the Hessian metric $\operatorname{Hess}(L)$ is non-singular.

A gauge-invariant Lagrangian $L \in C^{\infty}\left(J^{1} C\right)$ is said to be weakly regular if the Hessian metric Hess $(\tilde{L})$ of the function in (5.2) associated to $L$ according to Utiyama's theorem, is non-singular.

In terms of the coordinate system $\left(x^{i} ; R_{j k}^{\alpha}\right), j<k$, on $\bigwedge^{2} T^{*} M \otimes \operatorname{ad} P$ introduced in Section 5.1, this means

$$
\operatorname{det}\left(\frac{\partial^{2} \tilde{L}}{\partial R_{i j}^{\alpha} \partial R_{k l}^{\beta}}\right)_{\beta, k<l}^{\alpha, i<j} \neq 0 .
$$

Remark 5.5. The inequation (5.3) imposes a generic condition on $\tilde{L}$. In fact, most of gauge-invariant Lagrangians in the field theory satisfy the weak regularity condition (5.3); for example, the general Yang-Mills Lagrangian on the bundle of connections of a principal bundle $P \rightarrow M$ with semisimple Lie group $G$ over a pseudo-Riemannian manifold $(M, g)$ (even when constructed by using a non-degenerate adjoint-invariant pairing on the Lie algebra other than the Cartan-Killing pairing, see $[3,5])$ is weakly regular. More generally, any quadratic function as in (5.2), which is simultaneously invariant under the adjoint representation and under the action of the gauge group of the principal bundle of $g$-orthonormal linear frames, gives rise to a 
weakly regular Lagrangian, see [4]. Similarly, Born-Infeld Lagrangians (e.g., see $[15,16])$ are also weakly regular. We remark on the fact that some special Lagrangians are not weakly regular; for example, if we let the matter field vanish in the Seiberg-Witten Lagrangian (e.g., see [9]) then we obtain a Lagrangian on the bundle of connections, which is not weakly regular (basically, because it involves only the self-dual part of the curvature). Finally, we should also remark that non-gauge-invariant Lagrangians (in the sense of Section 5.1) may produce gauge-invariant actions, as the Chern-Simons Lagrangian. All of them are not considered below.

Given an arbitrary fibred manifold $p: E \rightarrow M$, we recall that the projection $p_{10}: J^{1} E \rightarrow E$ is endowed with an affine-bundle structure modelled over $T^{*} M \otimes_{E} V(p)=p^{*} T^{*} M \otimes V(p)$; see the item (b) in Section 2.3. In the particular case of the bundle of connections, which is itself an affine bundle modelled over $T^{*} M \otimes \operatorname{ad} P$, we conclude that $p_{10}: J^{1} C \rightarrow C$ is an affine bundle modelled over

$$
T^{*} M \otimes_{C} V(p)=p^{*}\left(\otimes^{2} T^{*} M \otimes \operatorname{ad} P\right) .
$$

Hence, sections of $T^{*} M \otimes_{C} V(p)$ can be considered as ad $P$-valued covariant tensors of degree 2 on $M$ with coefficients in $C$.

Theorem 5.6. Let $\overline{\mathcal{S}}_{\Lambda}$ (resp. $\mathcal{S}_{\Lambda}$ ) denote the set of solutions to H-C (resp. $E-L)$ equations of a weakly regular gauge-invariant Lagrangian $L$ on the bundle of connections $p: C \rightarrow M$ of a principal bundle $\pi: P \rightarrow M$. If $\bar{s}: M \rightarrow$ $J^{1} C$ belongs to $\overline{\mathcal{S}}_{\Lambda}$, then the section $s=p_{10} \circ \bar{s}$ belongs to $\mathcal{S}_{\Lambda}$. Hence, a natural projection exists $\varrho: \overline{\mathcal{S}}_{\Lambda} \rightarrow \mathcal{S}_{\Lambda}, \varrho(\bar{s})=p_{10} \circ \bar{s}$, which is an affine bundle modelled as follows:

$$
\varrho^{-1}(s)=\left\{j^{1} s+t: t \in \Gamma\left(S^{2} T^{*} M \otimes \operatorname{ad} P\right)\right\}, \quad \forall s \in \mathcal{S}_{\Lambda} .
$$

Proof. We begin with the first $\mathrm{H}-\mathrm{C}$ equation in Proposition 5.2. As $L=$ $\tilde{L} \circ \Omega$, we obtain

$$
\frac{\partial L}{\partial A_{i, i}^{\alpha}}=0, \quad \frac{\partial L}{\partial A_{i, k}^{\alpha}}=\frac{\partial \tilde{L}}{\partial R_{i k}^{\alpha}} \circ \Omega
$$

where we have set $R_{i k}^{\alpha}=-R_{k i}^{\alpha}$ for $i>k$. Hence,

$$
\left(s_{h, j}^{\beta}-\bar{s}_{h, j}^{\beta}\right)\left(\frac{\partial^{2} \tilde{L}}{\partial R_{i k}^{\alpha} \partial R_{h j}^{\beta}} \circ(\Omega \circ \bar{s})\right)=0 .
$$


If we assume the weak regularity condition (5.3) holds, then the previous equation yields

$$
\left(s_{h, j}^{\beta}-\bar{s}_{h, j}^{\beta}\right)-\left(s_{j, h}^{\beta}-\bar{s}_{j, h}^{\beta}\right)=0, \quad \forall \beta, h, j .
$$

If we write $\bar{s}=j^{1} s+t$, for a two-tensor $t$, the condition above means that $t$ is symmetric; that is, $t$ is a section of $S^{2} T^{*} M \otimes \operatorname{ad} P \rightarrow M$.

Next, we study the second equation in Proposition 5.2. Taking equations (5.5) into account, we have

$$
-\frac{\partial}{\partial x^{j}}\left(\frac{\partial L}{\partial A_{i, j}^{\alpha}} \circ \bar{s}\right)+\frac{\partial L}{\partial A_{i}^{\alpha}} \circ \bar{s}+\left(s_{h, j}^{\beta}-\bar{s}_{h, j}^{\beta}\right)\left(\frac{\partial}{\partial A_{i}^{\alpha}}\left(\frac{\partial \tilde{L}}{\partial R_{h j}^{\beta}} \circ \Omega\right) \circ \bar{s}\right)=0 .
$$

The last term vanishes identically as $s_{h, j}^{\beta}-\bar{s}_{h, j}^{\beta}$ is symmetric by virtue of (5.6) and we have $\partial \tilde{L} / \partial R_{h j}^{\beta}=-\partial \tilde{L} / \partial R_{j h}^{\beta}$. Then, the second equation reduces to

$$
-\frac{\partial}{\partial x^{j}}\left(\frac{\partial L}{\partial A_{i, j}^{\alpha}} \circ \bar{s}\right)+\frac{\partial L}{\partial A_{i}^{\alpha}} \circ \bar{s}=0,
$$

which is precisely the E-L equation, but evaluated at $\bar{s}$ instead of $j^{1} s$. Nevertheless, the following formula is readily checked:

$$
\frac{\partial L}{\partial A_{i}^{\alpha}}=2\left(c_{\alpha \gamma}^{\beta} A_{j}^{\gamma} \frac{\partial \tilde{L}}{\partial R_{i j}^{\beta}}\right) \circ \Omega
$$

Moreover, from (5.6) we deduce

$$
\begin{aligned}
\Omega \circ \bar{s} & =\left(\bar{s}_{i, j}^{\alpha}-\bar{s}_{j, i}^{\alpha}-c_{\beta \gamma}^{\alpha} s_{i}^{\beta} s_{j}^{\gamma}\right) d x^{i} \wedge d x^{j} \otimes \tilde{B}_{\alpha} \\
& =\left(s_{i, j}^{\alpha}-s_{j, i}^{\alpha}-c_{\beta \gamma}^{\alpha} s_{i}^{\beta} s_{j}^{\gamma}\right) d x^{i} \wedge d x^{j} \otimes \tilde{B}_{\alpha} \\
& =\Omega \circ j^{1} s .
\end{aligned}
$$

Therefore, from formulas (5.5) and (5.8), we conclude that equation (5.7) coincides with the E-L equation for $s$.

Remark 5.7. As mentioned in the Introduction, formula (5.9) shows that the curvature remains constant along the fibre of $\varrho$ over any $j^{1} s \in \mathcal{S}_{\Lambda}$. 
Corollary 5.8. The $H-C$ equations of a weakly regular gauge-invariant Lagrangian $L$ on the bundle of connections $p: C \rightarrow M$ of a principal bundle $\pi: P \rightarrow M$, are equivalent to the following system:

$$
\begin{gathered}
\left(s_{h, j}^{\alpha}-\bar{s}_{h, j}^{\alpha}\right)-\left(s_{j, h}^{\alpha}-\bar{s}_{j, h}^{\alpha}\right)=0, \quad \forall \alpha, h, j . \\
-\frac{\partial}{\partial x^{j}}\left(\frac{\partial L}{\partial A_{i, j}^{\alpha}} \circ j^{1} s\right)+\frac{\partial L}{\partial A_{i}^{\alpha}} \circ j^{1} s=0, \quad \forall \alpha, i,
\end{gathered}
$$

where $\bar{s}: M \rightarrow J^{1} C$ is a section of $p_{1}: J^{1} C \rightarrow M$, and $s_{i}^{\alpha}, s_{i, j}^{\alpha}$, and $\bar{s}_{i, j}^{\alpha}$ are as in Proposition 5.2.

Remark 5.9. For every section $\bar{s}: M \rightarrow J^{1} C$ of $p_{1}: J^{1} C \rightarrow M$, let $s: M \rightarrow$ $C$ be the section of $p: C \rightarrow M$ defined by $s=p_{10} \circ \bar{s}$. As the points $\bar{s}(x)$, $j_{x}^{1} s \in J^{1} C$ lie over the same fibre of $p_{10}: J^{1} C \rightarrow C$ and this map admits an affine-bundle structure modelled over the vector bundle (5.4), a map

$$
\delta_{C}: J^{1}\left(p_{1}\right) \rightarrow \otimes^{2} T^{*} M \otimes \operatorname{ad} P
$$

exists such that, $\delta_{C}\left(j_{x}^{1} \bar{s}\right)=\bar{s}(x)-j_{x}^{1} s$. If alt: $\otimes^{2} T^{*} M \otimes \operatorname{ad} P \rightarrow \wedge^{2} T^{*} M \otimes$ $\operatorname{ad} P$ denotes the anti-symmetrization operator, then the first group of $\mathrm{H}-\mathrm{C}$ equations in Corollary 5.8 means that $j^{1} \bar{s}$ takes values into the sub-bundle $\operatorname{ker}\left(\right.$ alt $\left.\circ \delta_{C}\right)$.

Proposition 5.10. If $\Lambda$ is a gauge-invariant Lagrangian density on the bundle of connections of a principal bundle $\pi: P \rightarrow M$ and $\bar{s} \in \overline{\mathcal{S}}_{\Lambda}$ (resp. $s \in$ $\left.\mathcal{S}_{\Lambda}\right)$, then $\Phi_{C}^{(1)} \circ \bar{s} \in \overline{\mathcal{S}}_{\Lambda}$ (resp. $\left.\Phi_{C} \circ s \in \mathcal{S}_{\Lambda}\right)$ for every $\Phi \in \mathrm{Gau} P$. Accordingly, the gauge group of $P$ acts (on the left) on $\overline{\mathcal{S}}_{\Lambda}$ (resp. $\mathcal{S}_{\Lambda}$ ) by setting $\Phi \cdot \bar{s}=\Phi_{C}^{(1)} \circ \bar{s}\left(r e s p . \Phi \cdot s=\Phi_{C} \circ s\right), \forall \bar{s} \in \overline{\mathcal{S}}_{\Lambda}\left(\right.$ resp. $\left.\forall s \in \mathcal{S}_{\Lambda}\right), \forall \Phi \in \mathrm{Gau} P$.

Proof. We prove that the section $\Phi_{C}^{(1)} \circ \bar{s}$ of $p_{1}$ is a solution to $\mathrm{H}-\mathrm{C}$ equation (3.1). For every $Z \in \mathfrak{X}^{v}\left(J^{1} E\right)$, we set

$$
Y=\left(\Phi_{C}^{(1)}\right)^{-1} \cdot Z \in \mathfrak{X}^{v}\left(J^{1} E\right) .
$$

As $\Lambda$ is gauge invariant, we have $\left(\Phi_{C}^{(1)}\right)^{*} \Lambda=\Lambda$, from the functorial character of the Poincaré-Cartan form (see [7]) we obtain

$$
\begin{aligned}
\left(\Phi_{C}^{(1)} \circ \bar{s}\right)^{*}\left(i_{Z} d \Theta_{\Lambda}\right) & =\bar{s}^{*}\left(\Phi_{C}^{(1)}\right)^{*}\left(i_{Z} d \Theta_{\Lambda}\right) \\
& =\bar{s}^{*} i_{Y} d\left(\left(\Phi_{C}^{(1)}\right)^{*} \Theta_{\Lambda}\right) \\
& =\bar{s}^{*} i_{Y} d \Theta\left(\Phi_{C}^{(1)}\right)^{*} \Lambda \\
& =\bar{s}^{*} i_{Y} d \Theta_{\Lambda} \\
& =0 .
\end{aligned}
$$


The proof for the solutions to $\mathrm{E}-\mathrm{L}$ equations, is similar and therefore it is omitted.

Theorem 5.6 shows that the set of solutions of $\mathrm{H}-\mathrm{C}$ trivially fibers over the set of solutions of E-L. On the other hand, for gauge-invariant problems, the moduli space of solutions under the action of the (restricted) gauge group plays a relevant role. We now study the relationship between the moduli of $\mathrm{H}-\mathrm{C}$ and $\mathrm{E}-\mathrm{L}$ showing that the first fibres over the second, but not necessarily in a trivial way. First, note that the gauge group GauP acts on the adjoint bundle ad $P$ by setting $\Phi_{\text {ad }}\left((u, B)_{\text {ad }}\right)=(\Phi(u), B)_{\text {ad }}, \forall \Phi \in$ $\operatorname{Gau} P, \forall(u, B) \in P \times \mathfrak{g}$, and this action obviously induces another action on $S^{2} T^{*} M \otimes \operatorname{ad} P$ as follows:

$$
\begin{aligned}
& \Phi_{\mathrm{ad} P}\left(w_{1} \odot w_{2} \otimes v\right)=w_{1} \odot w_{2} \otimes \Phi_{\mathrm{ad} P}(v), \\
& \forall w_{1}, w_{2} \in T_{x}^{*} M, \forall v \in(\operatorname{ad} P)_{x}
\end{aligned}
$$

where the symbol $\odot$ denotes symmetric product.

Proposition 5.11. Given a point $x_{0} \in M$, let $\mathrm{Gau}_{x_{0}} P$ be the subgroup of gauge transformations $\Phi \in \mathrm{Gau} P$ such that, $\Phi(u)=u, \forall u \in \pi^{-1}\left(x_{0}\right)$. Then

(i) For every gauge-invariant Lagrangian density $\Lambda$ on the bundle of connections of $\pi: P \rightarrow M$, the quotient map $\kappa_{P}: \mathcal{S}_{\Lambda} \rightarrow \mathcal{S}_{\Lambda} / \mathrm{Gau}_{x_{0}} P$ is a set-theoretical principal $\mathrm{Gau}_{x_{0}} P$-bundle.

(ii) In addition, if $\Lambda$ is weakly regular, the projection $\varrho: \overline{\mathcal{S}}_{\Lambda} \rightarrow \mathcal{S}_{\Lambda}$ defined in Theorem 5.6, induces a mapping

$$
\begin{aligned}
& \varrho_{\operatorname{Gau}_{x_{0}} P}: \overline{\mathcal{S}}_{\Lambda} / \operatorname{Gau}_{x_{0}} P \rightarrow \mathcal{S}_{\Lambda} / \operatorname{Gau}_{x_{0}} P, \\
& \varrho_{\operatorname{Gau}_{x_{0}} P}\left(\bar{s} \bmod \operatorname{Gau}_{x_{0}} P\right)=\varrho(\bar{s}) \bmod \operatorname{Gau}_{x_{0}} P,
\end{aligned}
$$

which is the vector bundle associated to the principal bundle $\kappa_{P}$ by the action on $S^{2} \Omega^{1}(M) \otimes \operatorname{gau} P$ induced on the sections of $S^{2} T^{*} M \otimes \operatorname{ad} P$ by the action of $\mathrm{Gau} P$ defined in the formula (5.10) above.

Proof. As is known, $\mathrm{Gau}_{x_{0}} P$ acts freely on the space of connections, i.e., on the sections of $p: C \rightarrow M$ and, in particular, on $\overline{\mathcal{S}}_{\Lambda}$ and on $\mathcal{S}_{\Lambda}$ (e.g., see [6, Theorem 2.2.4], [13, III.C]). Nevertheless, the quotients $\overline{\mathcal{S}}_{\Lambda} / \mathrm{Gau}_{x_{0}} P$ and $\mathcal{S}_{\Lambda} / \mathrm{Gau}_{x_{0}} P$ may be singular, e.g., see [6, p. 134]. Because of this, we consider such structure from the set-theoretical point of view only. 
Furthermore, the mapping $\varrho_{\mathrm{Gau}_{x_{0}} P}$ is well defined as

$$
\begin{aligned}
\varrho\left(\Phi_{C}^{(1)} \circ \bar{s}\right) & =\left(p_{10} \circ \Phi_{C}^{(1)}\right) \circ \bar{s} \\
& =\left(\Phi_{C} \circ p_{10}\right) \circ \bar{s} \\
& =\Phi_{C} \circ \varrho(\bar{s}),
\end{aligned}
$$

and from Theorem 5.6 it follows that every $\bar{s} \in \overline{\mathcal{S}}_{\Lambda}$ can be uniquely written as $\bar{s}=j^{1} s+t$, where $s=\varrho(\bar{s})$ and $t \in S^{2} \Omega^{1}(M) \otimes$ gau $P$. Hence, $\bar{s}$ can be identified to the pair $(s, t)$, i.e., $\overline{\mathcal{S}}_{\Lambda} \cong \mathcal{S}_{\Lambda} \times S^{2} \Omega^{1}(M) \otimes$ gau $P$. Recalling that $\Phi_{C}: C \rightarrow C$ is an affine-bundle morphism whose associated vector bundle is $\operatorname{id}_{T^{*} M} \otimes \Phi_{\mathrm{ad}}: T^{*} M \otimes \operatorname{ad} P \rightarrow T^{*} M \otimes \operatorname{ad} P$, we have

$$
\begin{aligned}
\Phi_{C}^{(1)} \circ \bar{s} & =\Phi_{C}^{(1)} \circ\left(j^{1} s+t\right) \\
& =J^{1}\left(\Phi_{C} \circ s\right)+\Phi_{\mathrm{ad}} \cdot t,
\end{aligned}
$$

thus concluding the proof.

Theorem 5.12. With the same notations as in Section 4 and the same assumptions as in Theorem 5.6, if $\bar{X} \in T_{\bar{s}} \overline{\mathcal{S}}_{\Lambda}$, then $\left(p_{10}\right)_{*} \circ \bar{X} \in T_{s} \mathcal{S}_{\Lambda}$, where $s=p_{10} \circ \bar{s}$. Hence, the natural map $\varrho: \overline{\mathcal{S}}_{\Lambda} \rightarrow \mathcal{S}_{\Lambda}$ induces a linear map $\varrho_{*}: T_{\bar{s}} \overline{\mathcal{S}}_{\Lambda} \rightarrow T_{s} \mathcal{S}_{\Lambda}, \varrho_{*}(\bar{X})=\left(p_{10}\right)_{*} \circ \bar{X}$. Moreover, $\operatorname{ker} \varrho_{*} \cong \Gamma\left(S^{2} T^{*} M \otimes \operatorname{ad} P\right)$.

Proof. We first begin with the second Jacobi equation (4.4) for a gaugeinvariant Lagrangian $L: J^{1} C \rightarrow \mathbb{R}$ and a Jacobi vector field

$$
\bar{X}=v_{i}^{\alpha} \frac{\partial}{\partial A_{i}^{\alpha}}+v_{i, j}^{\alpha} \frac{\partial}{\partial A_{i, j}^{\alpha}}, \quad v_{i}^{\alpha}, v_{i, j}^{\alpha} \in C^{\infty}(M),
$$

along a solution $\bar{s} \in \overline{\mathcal{S}}_{\Lambda}$. We have

$$
\begin{aligned}
0= & \left(\frac{\partial^{2} L}{\partial A_{r, i}^{\alpha} \partial A_{h, j}^{\sigma}} \circ \bar{s}\right)\left(\frac{\partial v_{r}^{\alpha}}{\partial x^{i}}-v_{r, i}^{\alpha}\right) \\
& +\left(\frac{\partial s_{r}^{\alpha}}{\partial x^{i}}-\bar{s}_{r, i}^{\alpha}\right)\left(\frac{\partial^{3} L}{\partial A_{l}^{\beta} \partial A_{r, i}^{\alpha} \partial A_{h, j}^{\sigma}} \circ \bar{s}\right) v_{l}^{\beta} \\
& +\left(\frac{\partial s_{r}^{\alpha}}{\partial x^{i}}-\bar{s}_{r, i}^{\alpha}\right)\left(\frac{\partial^{3} L}{\partial A_{r, i}^{\alpha} \partial A_{l, k}^{\beta} \partial A_{h, j}^{\sigma}} \circ \bar{s}\right) v_{l, k}^{\beta},
\end{aligned}
$$

for any $\sigma, h, j$. From Theorem $5.6, j^{1} s-\bar{s}$ is a symmetric tensor. Moreover, taking the formula (5.5) into account, the last two summands of (5.11) 
vanish. We thus obtain

$$
0=\left(\frac{\partial^{2} L}{\partial A_{r, i}^{\alpha} \partial A_{s, j}^{\sigma}} \circ \bar{s}\right)\left(\frac{\partial v_{r}^{\alpha}}{\partial x^{i}}-v_{r, i}^{\alpha}\right)
$$

which, assuming the weak regularity of $L$, implies

$$
v_{r, i}^{\alpha}=\frac{\partial v_{r}^{\alpha}}{\partial x^{i}}+t_{r, i}^{\alpha}
$$

where $t_{r, i}^{\alpha}$ are the components of a symmetric tensor $t \in \Gamma\left(S^{2} T^{*} M \otimes \operatorname{ad} P\right)$. From formula (2.1) and Lemma 2.1 we conclude $\bar{X}=X^{(1)}+t$, where $X^{(1)}$ is the one-jet prolongation of the vector field along $s$ given by $X=v_{i}^{\alpha} \partial / \partial A_{i}^{\alpha}$.

Next, we consider the first Jacobi equation (4.3), from which we obtain

$$
\begin{aligned}
& 0=\left(\frac{\partial^{2} L}{\partial A_{r}^{\alpha} \partial A_{q}^{\sigma}} \circ \bar{s}-\frac{\partial^{3} L}{\partial x^{i} \partial A_{r}^{\alpha} \partial A_{q, i}^{\sigma}} \circ \bar{s}+\left(s_{t, i}^{\beta}-\bar{s}_{t, i}^{\beta}\right)\left(\frac{\partial^{3} L}{\partial A_{r}^{\alpha} \partial A_{q}^{\sigma} \partial A_{t, i}^{\beta}} \circ \bar{s}\right)\right. \\
& \left.-s_{t, j}^{\gamma}\left(\frac{\partial^{3} L}{\partial A_{r}^{\alpha} \partial A_{t}^{\gamma} \partial A_{q, j}^{\sigma}} \circ \bar{s}\right)-\frac{\partial \bar{s}_{t, h}^{\gamma}}{\partial x^{j}}\left(\frac{\partial^{3} L}{\partial A_{r}^{\alpha} \partial A_{t, h}^{\gamma} \partial A_{q, j}^{\sigma}} \circ \bar{s}\right)\right) v_{r}^{\alpha} \\
& +\left(\frac{\partial^{2} L}{\partial A_{q}^{\sigma} \partial A_{r, i}^{\alpha}} \circ \bar{s}-\frac{\partial^{2} L}{\partial A_{r}^{\alpha} \partial A_{q, i}^{\sigma}} \circ \bar{s}\right) \frac{\partial v_{r}^{\alpha}}{\partial x^{i}}-\left(\frac{\partial^{2} L}{\partial A_{r, j}^{\alpha} \partial A_{q, i}^{\sigma}} \circ \bar{s}\right) \frac{\partial v_{r, j}^{\alpha}}{\partial x^{i}} \\
& +\left(\left(s_{t, j}^{\beta}-\bar{s}_{t, j}^{\beta}\right)\left(\frac{\partial^{3} L}{\partial A_{q}^{\sigma} \partial A_{r, i}^{\alpha} \partial A_{t, j}^{\beta}} \circ \bar{s}\right)-\frac{\partial^{3} L}{\partial x^{j} \partial A_{r, i}^{\alpha} \partial A_{q, j}^{\sigma}} \circ \bar{s}\right. \\
& \left.-s_{t, j}^{\beta}\left(\frac{\partial^{3} L}{\partial A_{t}^{\beta} \partial A_{r, i}^{\alpha} \partial A_{q, j}^{\sigma}} \circ \bar{s}\right)-\frac{\partial \bar{s}_{t, h}^{\beta}}{\partial x^{j}}\left(\frac{\partial^{3} L}{\partial A_{t, h}^{\beta} \partial A_{r, i}^{\alpha} \partial A_{j}^{\sigma}} \circ \bar{s}\right)\right) v_{r, i}^{\alpha} .
\end{aligned}
$$

Again taking the symmetry of the differences $s_{t, i}^{\beta}-\bar{s}_{t, i}^{\beta}$ (and their derivatives) into account, the previous equation reduces to the following:

$$
\begin{aligned}
0= & \left(\frac{\partial^{2} L}{\partial A_{r}^{\alpha} \partial A_{q}^{\sigma}} \circ \bar{s}-\frac{\partial^{3} L}{\partial x^{i} \partial A_{r}^{\alpha} \partial A_{q, i}^{\sigma}} \circ \bar{s}-s_{t, j}^{\gamma}\left(\frac{\partial^{3} L}{\partial A_{r}^{\alpha} \partial A_{t}^{\gamma} \partial A_{q, j}^{\sigma}} \circ \bar{s}\right)\right. \\
& \left.-\frac{\partial^{2} s_{t}^{\gamma}}{\partial x^{h} \partial x^{j}}\left(\frac{\partial^{3} L}{\partial A_{r}^{\alpha} \partial A_{t, h}^{\gamma} \partial A_{q, j}^{\sigma}} \circ \bar{s}\right)\right) v_{r}^{\alpha} \\
& +\left(\frac{\partial^{2} L}{\partial A_{q}^{\sigma} \partial A_{r, i}^{\alpha}} \circ \bar{s}-\frac{\partial^{2} L}{\partial A_{r}^{\alpha} \partial A_{q, i}^{\sigma}} \circ \bar{s}\right) \frac{\partial v_{r}^{\alpha}}{\partial x^{i}}-\left(\frac{\partial^{2} L}{\partial A_{r, j}^{\alpha} \partial A_{q, i}^{\sigma}} \circ \bar{s}\right) \frac{\partial v_{r, j}^{\alpha}}{\partial x^{i}}
\end{aligned}
$$




$$
\begin{aligned}
& -\left(\frac{\partial^{3} L}{\partial x^{j} \partial A_{r, i}^{\alpha} \partial A_{q, j}^{\sigma}} \circ \bar{s}+s_{t, j}^{\beta}\left(\frac{\partial^{3} L}{\partial A_{t}^{\beta} \partial A_{r, i}^{\alpha} \partial A_{q, j}^{\sigma}} \circ \bar{s}\right)\right. \\
& \left.+\frac{\partial^{2} s_{t}^{\beta}}{\partial x^{h} \partial x^{j}}\left(\frac{\partial^{3} L}{\partial A_{t, h}^{\beta} \partial A_{r, i}^{\alpha} \partial A_{q, j}^{\sigma}} \circ \bar{s}\right)\right) v_{r, i}^{\alpha} .
\end{aligned}
$$

By taking derivatives in (5.12), we obtain

$$
\frac{\partial v_{r, j}^{\alpha}}{\partial x^{i}}=\frac{\partial v_{r}^{\alpha}}{\partial x^{j} \partial x^{i}}+\frac{\partial t_{r, j}^{\alpha}}{\partial x^{i}},
$$

and substituting these expressions into equation (5.14), again by virtue of the skew-symmetry of $\partial L / \partial A_{i, j}^{\alpha}$, we have

$$
\begin{aligned}
0= & \left(\frac{\partial^{2} L}{\partial A_{r}^{\alpha} \partial A_{q}^{\sigma}} \circ \bar{s}-\frac{\partial^{3} L}{\partial x^{i} \partial A_{r}^{\alpha} \partial A_{q, i}^{\sigma}} \circ \bar{s}-s_{t, j}^{\gamma}\left(\frac{\partial^{3} L}{\partial A_{r}^{\alpha} \partial A_{t}^{\gamma} \partial A_{q, j}^{\sigma}} \circ \bar{s}\right)\right. \\
& \left.-\frac{\partial^{2} s_{t}^{\gamma}}{\partial x^{j} \partial x^{h}}\left(\frac{\partial^{3} L}{\partial A_{r}^{\alpha} \partial A_{t, h}^{\gamma} \partial A_{q, j}^{\sigma}} \circ \bar{s}\right)\right) v_{r}^{\alpha} \\
& +\left(\frac{\partial^{2} L}{\partial A_{q}^{\sigma} \partial A_{r, i}^{\alpha}} \circ \bar{s}-\frac{\partial^{2} L}{\partial A_{r}^{\alpha} \partial A_{q, i}^{\sigma}} \circ \bar{s}\right) \frac{\partial v_{r}^{\alpha}}{\partial x^{i}}-\left(\frac{\partial^{2} L}{\partial A_{r, j}^{\alpha} \partial A_{q, i}^{\sigma}} \circ \bar{s}\right) \frac{\partial v_{r}^{\alpha}}{\partial x^{i} \partial x^{j}} \\
& -\left(\frac{\partial^{3} L}{\partial x^{j} \partial A_{r, i}^{\alpha} \partial A_{q, j}^{\sigma}} \circ \bar{s}+s_{t, j}^{\beta}\left(\frac{\partial^{3} L}{\partial A_{t}^{\beta} \partial A_{r, i}^{\alpha} \partial A_{q, j}^{\sigma}} \circ \bar{s}\right)\right. \\
& \left.+\frac{\partial^{2} s_{t}^{\beta}}{\partial x^{h} \partial x^{j}}\left(\frac{\partial^{3} L}{\partial A_{t, h}^{\beta} \partial A_{r, i}^{\alpha} \partial A_{q, j}^{\sigma}} \circ \bar{s}\right)\right) \frac{\partial v_{r}^{\alpha}}{\partial x^{i}} .
\end{aligned}
$$

Finally, taking the equations $\Omega \circ \bar{s}=\Omega \circ j^{1} s, L=\bar{L} \circ \Omega$, into account, we conclude that $\bar{s}$ can be replaced by $j^{1} s$ into the previous equation and we can end the proof by simply applying Remark 4.6.

Corollary 5.13. A Jacobi field $\bar{X} \in T_{\bar{S}} \overline{\mathcal{S}}_{\Lambda}$ is integrable if and only if the Jacobi field $\varrho_{*}(\bar{X})=X \in T_{s} \mathcal{S}_{\Lambda}, s=p_{10} \circ \bar{s}$, is integrable.

Proof. If $\bar{X} \in T_{\bar{s}} \overline{\mathcal{S}}_{\Lambda}$ is an integrable Jacobi field, then $\bar{X}=d /\left.d \varepsilon\right|_{\varepsilon=0} \bar{s}_{\varepsilon}$ where $\bar{s}_{\varepsilon}=j^{1} s_{\varepsilon}+t_{\varepsilon}$, with $\pi_{10} \circ \bar{s}=s_{\varepsilon} \in \mathcal{S}_{\Lambda}, t_{\varepsilon}$ being a symmetric tensor. Then,

$$
\varrho_{*}(\bar{X})=\left(\pi_{10}\right)_{*} \bar{X}=\left.\frac{d}{d \varepsilon}\right|_{\varepsilon=0} \pi_{10} \circ \bar{s}_{\varepsilon}=\left.\frac{d}{d \varepsilon}\right|_{\varepsilon=0} s_{\varepsilon} .
$$


Hence $X=\varrho_{*}(\bar{X})$ is integrable. Conversely, assume $X=d /\left.d \varepsilon\right|_{\varepsilon=0} s_{\varepsilon}, s_{\epsilon} \in$ $\mathcal{S}_{\Lambda}$, and $\bar{X} \in\left(\varrho_{*}\right)^{-1} X$. We know that $\bar{X}=X^{(1)}+t$, where $t$ is a symmetric tensor. Then, $\bar{X}=d /\left.d \varepsilon\right|_{\varepsilon=0} \bar{s}_{\varepsilon}$ with $\bar{s}_{\varepsilon}=j^{1} s_{\varepsilon}+\varepsilon t \in \overline{\mathcal{S}}_{\Lambda}$, and $\bar{X}$ is integrable.

\section{$6 \quad \mathrm{H}-\mathrm{C}$ self-dual and anti-self-dual connections}

Let $(M, g)$ be a pseudo-Riemannian $n$-dimensional oriented connected manifold of signature $\left(n^{+}, n^{-}\right), n=n^{+}+n^{-}$, and let $\mathbf{v}_{g}=\sqrt{\left|\operatorname{det}\left(g_{i j}\right)\right|} d x^{1} \wedge$ $\cdots \wedge d x^{n}, g_{i j}=g\left(\partial / \partial x^{i}, \partial / \partial x^{j}\right)$, be its pseudo-Riemannian volume form. The canonical duality isomorphism attached to $g$ is denoted by $T_{x} M \rightarrow$ $T_{x}^{*} M, X \mapsto X^{b}$, with inverse map $T_{x}^{*} M \rightarrow T_{x} M, w \mapsto w^{\sharp}$. Let $g^{(r)}$ be the metric on $\wedge^{r} T^{*} M$ given by $g^{(r)}\left(w^{1} \wedge \cdots \wedge w^{r}, \bar{w}^{1} \wedge \cdots \wedge \bar{w}^{r}\right)=\operatorname{det}\left(g\left(\left(w^{i}\right)^{\sharp}\right.\right.$, $\left.\left.\left(\bar{w}^{j}\right)^{\sharp}\right)\right)_{i, j=1}^{r}$.

Let $V \rightarrow M$ be a vector bundle. The Hodge star can be extended to $V$-valued forms as follows: $\star\left(\omega_{r} \otimes v\right)=\left(\star \omega_{r}\right) \otimes v, \forall \omega_{r} \in \Lambda^{r} T_{x}^{*} M, \forall v \in V_{x}$.

Let $\pi: P \rightarrow M$ be a principal $G$-bundle and let $\pi_{\mathrm{ad} P}: \operatorname{ad} P \rightarrow M$ be the adjoint bundle; i.e., the bundle associated with $P$ under the adjoint representation of $G$ on its Lie algebra $\mathfrak{g}$. For every $B \in \mathfrak{g}$ and every $u \in P$, let $(u, B)_{G}$ be the coset of $(u, B) \in P \times \mathfrak{g}$ modulo $G$. A symmetric bilinear form $\langle\cdot, \cdot\rangle \in S^{2} \mathfrak{g}^{*}$ is said to be invariant under the adjoint representation if the following equation holds: $\left\langle\operatorname{Ad}_{g} B, \operatorname{Ad}_{g} C\right\rangle=\langle B, C\rangle, \forall g \in G, \forall B, C \in \mathfrak{g}$. By taking derivatives on this equation we obtain $\langle[A, B], C\rangle+\langle B,[A, C]\rangle=0$, $\forall A, B, C \in \mathfrak{g}$. If the group $G$ is connected, then both equations above are equivalent.

Every symmetric bilinear form $\langle\cdot, \cdot\rangle \in S^{2} \mathfrak{g}^{*}$ invariant under the adjoint representation induces a fibred metric $\langle\langle\cdot, \cdot\rangle\rangle: \operatorname{ad} P \oplus \operatorname{ad} P \rightarrow \mathbb{R}$ by setting

$$
\left\langle\left\langle(u, B)_{G},(u, C)_{G}\right\rangle\right\rangle=\langle B, C\rangle, \quad \forall u \in P, \forall B, C \in \mathfrak{g} .
$$

We further assume that the pairing (6.1) is non-degenerate.

Every pseudo-Riemannian metric $g$ on $M$ and every fibred $\langle\langle\cdot, \cdot\rangle\rangle$ on ad $P$ induce a fibred metric on the vector bundle of ad $P$-valued differential $r$-forms on $M$ as follows: $\left(\left(\alpha_{r} \otimes a, \beta_{r} \otimes b\right)\right)=g^{(r)}\left(\alpha_{r}, \beta_{r}\right)\langle\langle a, b\rangle\rangle, \forall \alpha_{r}, \beta_{r} \in \Lambda^{r} T_{x}^{*} M$, and $\forall a, b \in(\operatorname{ad} P)_{x}$. Moreover, the pairing (6.1) defines an exterior product (see [1]),

$$
\begin{aligned}
& \dot{\wedge}:\left(\bigwedge^{\bullet} T^{*} M \otimes \operatorname{ad} P\right) \oplus\left(\bigwedge^{\bullet} T^{*} M \otimes \operatorname{ad} P\right) \rightarrow \bigwedge^{\bullet} T^{*} M, \\
& \left(\alpha_{q} \otimes a\right) \dot{\wedge}\left(\beta_{r} \otimes b\right)=\left(\alpha_{q} \wedge \beta_{r}\right)\langle\langle a, b\rangle .
\end{aligned}
$$


Let $p: C \rightarrow M$ be the bundle of connections of $P$. According to the previous definitions, a pseudo-Riemannian metric $g$ on $M$ and an adjointinvariant symmetric bilinear form $\langle\cdot, \cdot\rangle$ allow one to define a quadratic Lagrangian density $\Lambda=L \mathbf{v}_{g}$ on $J^{1} C$ by setting,

$$
\begin{aligned}
\Lambda\left(j_{x}^{1} s\right) & =\left(\left(\Omega^{s}(x), \Omega^{s}(x)\right)\right) \mathbf{v}_{g}(x) \\
& =\Omega^{s}(x) \dot{\wedge} \star \Omega^{s}(x),
\end{aligned}
$$

where $s$ is a local section of $p$ defining a principal connection whose curvature form is denoted by $\Omega^{s}$. In [3] it is proved that the E-L equations of the Lagrangians above are seen to be independent of the pairing (6.1) and they coincide with the classical Yang-Mills equations: $\nabla^{s} \star \Omega^{s}=0$.

Theorem 6.1. In addition to the hypotheses above, assume $\operatorname{dim} M=4$ and $g$ is a Riemannian metric. Let $\mathcal{S}_{\Lambda}^{+}$(resp. $\mathcal{S}_{\Lambda}^{-}$) be the set of self-dual (resp. anti-self-dual) connections with respect to the Lagrangian density (6.2). A section $\bar{s}$ of $p_{1}: J^{1} C \rightarrow M$ belongs to the fibre $\varrho^{-1}(s)$, with $s \in \mathcal{S}_{\Lambda}^{+}$ (resp. $s \in \mathcal{S}_{\Lambda}^{-}$) if and only if the following equations hold:

$$
\begin{aligned}
\operatorname{alt}\left(\bar{s}-j^{1} s\right) & =0, \\
\star(\Omega \circ \bar{s}) & =(\Omega \circ \bar{s}) \quad(\text { resp. } \star(\Omega \circ \bar{s})=-(\Omega \circ \bar{s})),
\end{aligned}
$$

where alt: $\otimes^{2} T^{*} M \otimes \operatorname{ad} P \rightarrow \bigwedge^{2} T^{*} M \otimes \operatorname{ad} P$ is the alternating operator.

Remark 6.2. Equation (6.3) is a first-order differential equation, whereas (6.4) is a purely algebraic equation. The condition $\operatorname{alt}\left(\bar{s}-j^{1} s\right)=0$ is not specific of the Yang-Mills Lagrangian but general for any weakly regular gauge-invariant Lagrangian. In fact, it defines the subset of $\Gamma\left(p_{1}\right)$ given by

$$
\left\{j^{1} s+t: s \in \Gamma(p), t \in \Gamma\left(S^{2} T^{*} M \otimes \operatorname{ad} P\right)\right\} .
$$

It is thus interesting to note that the group of equations really defined by selfdual (resp. anti-self-dual) connections, is not longer a differential equation but an algebraic constrain on the subset (6.5).

Proof of Theorem 6.1. As $L$ is weakly regular, if $\bar{s} \in \overline{\mathcal{S}}_{\Lambda}$, then $\operatorname{alt}\left(\bar{s}-j^{1} s\right)=$ 0 by virtue of Remark 5.9. Moreover, as

$$
-\frac{\partial}{\partial x^{j}}\left(\frac{\partial L}{\partial A_{i, j}^{\alpha}} \circ \bar{s}\right)+\frac{\partial L}{\partial A_{i}^{\alpha}} \circ \bar{s}=-\frac{\partial}{\partial x^{j}}\left(\frac{\partial L}{\partial A_{i, j}^{\alpha}} \circ j^{1} s\right)+\frac{\partial L}{\partial A_{i}^{\alpha}} \circ j^{1} s,
$$


as $\Omega \circ \bar{s}=\Omega \circ j^{1} s$, we also obtain $\nabla^{s} \star \Omega^{s}=0$, where $\Omega^{s}=\Omega \circ j^{1} s$ and $s=p_{10} \circ \bar{s}$. Hence, the definition of a self-dual connection (i.e., $\star\left(\Omega \circ j^{1} s\right)=$ $\left.\left(\Omega \circ j^{1} s\right)\right)$ can be written as $\star(\Omega \circ \bar{s})=(\Omega \circ \bar{s})$ for every $\bar{s} \in \varrho^{-1}\left(\mathcal{S}_{\Lambda}^{+}\right)$. Similarly for $\mathcal{S}_{\Lambda}^{-}$.

\section{Acknowledgments}

Supported by Ministerio de Ciencia e Innovación of Spain under grants numbers MTM2011-22528 and MTM2010-19111.

\section{References}

[1] D. Bleecker, Gauge theory and variational principles, Global Analysis Pure and Applied Series A, 1, Addison-Wesley Publishing Co., Reading, MA, 1981.

[2] M. Castrillón López and J. Muñoz Masqué, The geometry of the bundle of connections, Math. Z. 236 (2001), 797-811.

[3] M. Castrillón López and J. Muñoz Masqué, Independence of Yang-Mills equations with respect to the invariant pairing in the Lie algebra, Int. J. Theor. Phys. 46(4) (2007), 1020-1026.

[4] M. Castrillón López and J. Muñoz Masqué, Gauge-invariant characterization of Yang-Mills-Higgs equations, Ann. Henri Poincaré 8 (2007), 203-217.

[5] M. Castrillón López, J. Muñoz Masqué and T. Ratiu, Gauge invariance and variational trivial problems on the bundle of connections, Differ. Geom. Appl. 19(2) (2003), 127-145.

[6] P. Cotta-Ramusino and C. Reina, The action of the group of bundleautomorphisms on the space of connections and the geometry of gauge theories, J. Geom. Phys. 1(3) (1984), 121-155.

[7] P.L. García Pérez, The Poincaré-Cartan invariant in the calculus of variations, Symposia Mathematica, Volume XIV (Convegno di Geometria Simplettica e Fisica Matematica, INDAM, Rome, 1973), London, Academic Press, 1974, 219-246.

[8] H. Goldschmidt and S. Sternberg, The Hamilton-Cartan formalism in the calculus of variations, Ann. Inst. Fourier (Grenoble) 23 (1973), 203-267.

[9] J. Jost, X. Peng and G. Wang, Variational aspects of the Seiberg-Witten functional, Calc. Var. Partial Differ. Equ. 4(3) (1996), 205-218. 
[10] S. Kobayashi and K. Nomizu, Foundations of Differential Geometry, New York, John Wiley \& Sons, Inc. (Interscience Division), Vol I, 1963.

[11] L. Lemaire and J.C. Wood, Jacobi fields along harmonic 2-spheres in $\mathbb{C} P^{2}$ are integrable, J. Lond. Math. Soc. (2) 66(2) (2002), 468-486.

[12] F. Lin and Ch. Wang, The analysis of harmonics maps and their heat lows, Singapore, World Scientific Publishing Co. Pte. Ltd., 2008.

[13] P.K. Mitter and C.M. Viallet, On the bundle of connections and the gauge orbit manifold in Yang-Mills theory, Comm. Math. Phys. 79(4) (1981), 457-472.

[14] J. Muñoz Masqué and L.M. Pozo Coronado, Parameter invariance in field theory and the Hamiltonian formalism, Fortschr. Phys. 48 (2000), 361-405.

[15] Y. Okawa, Derivative corrections to Dirac-Born-Infeld Lagrangian and non-commutative gauge theory, Nucl. Phys. B 566(1-2) (2000), 348-362.

[16] T. Suzuki, Born-Infeld action in $(n+2)$-dimension, the field equation and a soliton solution, Lett. Math. Phys. 47(2) (1999), 159-171. 
